\title{
Spallation-based neutron target for direct studies of neutron-induced reactions in inverse kinematics
}

\author{
René Reifarth, Kathrin Göbel, Tanja Heftrich, and Mario Weigand \\ Goethe-Universität Frankfurt, Frankfurt am Main, 60438 Frankfurt, Germany \\ Beatriz Jurado \\ CENBG, 33175 Gradignan, France \\ Franz Käppeler \\ Karlsruhe Institute of Technology, 76131 Karlsruhe, Germany \\ Yuri A. Litvinov \\ GSI Helmholtzzentrum für Schwerionenforschung, 64291 Darmstadt, Germany
}

(Received 29 November 2016; published 6 April 2017)

\begin{abstract}
We discuss the possibility to build a neutron target for nuclear reaction studies in inverse kinematics utilizing a storage ring and radioactive ion beams. The proposed neutron target is a specially designed spallation target surrounded by a large moderator of heavy water $\left(\mathrm{D}_{2} \mathrm{O}\right)$. We present the resulting neutron spectra and their properties as a target. We discuss possible realizations at different experimental facilities.
\end{abstract}

DOI: 10.1103/PhysRevAccelBeams.20.044701

\section{INTRODUCTION}

Neutron capture cross sections of unstable isotopes are important for neutron induced nucleosynthesis as well as for technological applications. The traditional time-offlight method [1] reaches its limits once the necessary detection of the reaction products is hampered by the size (mass) of the sample. Several factors may limit the sample mass: (a) The decay properties of radioactive isotopes interfere with the signals from the neutron capture or neutron-induced fission reactions [2]; (b) the limited range of charged reaction products requires a thin sample. In both cases, an increased neutron fluence at the sample position with ever improved neutron sources overcomes the lack of reaction rate [3-5].

Reference [6] proposed a combination of a radioactive beam facility, an ion storage ring and a high flux reactor to allow direct measurements of neutron-induced reactions over a wide energy range on isotopes with half-lives down to minutes. The authors discussed specific reactions, detection techniques and counting rates. Here, we present the possibility to replace the rather demanding reactor with a specially designed spallation neutron source. Figure 1 shows a sketch of the proposed setup.

\footnotetext{
*reifarth@physik.uni-frankfurt.de
}

Published by the American Physical Society under the terms of the Creative Commons Attribution 4.0 International license. Further distribution of this work must maintain attribution to the author(s) and the published article's title, journal citation, and DOI.
The advantages of such a setup over the reactor approach are manifold: No critical assembly is required, and therefore, the safety and security regulations are much less stringent. No actinides at all are used or produced. In particular, no minor actinides are produced avoiding long-lived

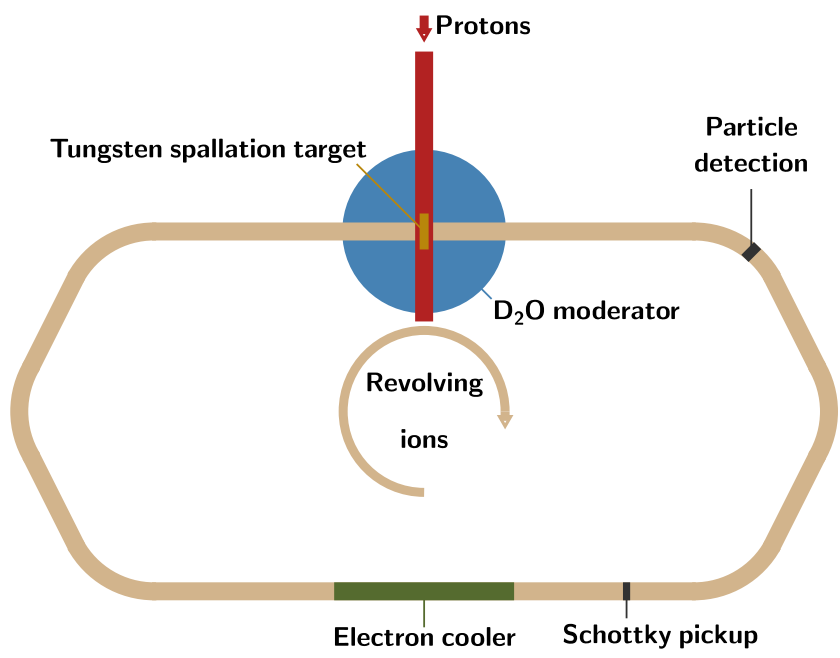

FIG. 1. Rough sketch of the proposed setup. Neutrons are produced by protons impinging on a tungsten spallation target (brown). The proton beam pipe (red) is orientated perpendicular to the ion beam pipe (light brown). The beam pipes do not intersect. The neutrons produced in the spallation process are moderated in the surrounding heavy water (blue). They penetrate the ion beam pipe and act as a neutron target for the ions. The ion beam pipe is part of a storage ring outside the moderator. The storage ring may contain additional equipment like an electron cooler (green), Schottky pickups and particle detectors (gray). 
radioactive waste. Last but not least, there are considerably less $\gamma$-rays per neutron.

Similar neutron densities as in a research reactor can be reached in a close-by ion beam pipe if the spallation target is surrounded by a moderator of heavy water $\left(\mathrm{D}_{2} \mathrm{O}\right)$. We present the concept in Sec. II and the corresponding simulations in Sec. III. It is feasible to build such a neutron target at facilities like LANSCE at LANL (USA), n_TOF/ ISOLDE at CERN (Switzerland), GSI/FAIR (Germany), HIRFL-CSR/HIAF (China), and others. We discuss the possible realizations in Sec. IV.

\section{CONCEPT AND GEOMETRY}

The center of the simulated setup is a tungsten spallation target, see Fig. 2. The cylindrical target is mounted inside an evacuated proton beam pipe with a radius of $2.5 \mathrm{~cm}$ and aligned in the direction of the proton beam. The protons impinge on the tungsten and produce neutrons. The material of the beam pipe has to be chosen such that it has only minor effects on the neutrons. The neutrons are moderated outside the proton beam pipe by heavy water in a surrounding sphere. A second (ion) beam pipe is orientated perpendicular to the proton beam pipe. The two pipes do not intersect since the ion beam pipe is shifted by $x=7.5 \mathrm{~cm}$ off the center of the setup. The neutrons penetrate into the ion beam pipe and serve as a target for the ions.

Compared to other elements, tungsten provides a high density of about $19 \mathrm{~g} \mathrm{~cm}^{-3}$ combined with a very high melting point of about $3,700 \mathrm{~K}$. We investigated two different tungsten target sizes, the "small version" with a radius of $1.5 \mathrm{~cm}$ and a length of $10 \mathrm{~cm}$, and the "large version" with a radius of $2.5 \mathrm{~cm}$ and length of $50 \mathrm{~cm}$.

We chose heavy-water moderators of different sizes with radii $0.5,1.0$, and $2.0 \mathrm{~m}$. If not specified otherwise, the center of the spherical moderator is also the center of the spallation target. A technical realization would require a cooling of the spallation target. The cooling should be realized with heavy water to avoid changes in the neutron physics investigated here. Most neutrons eventually escape the moderator volume after moderation and have to be
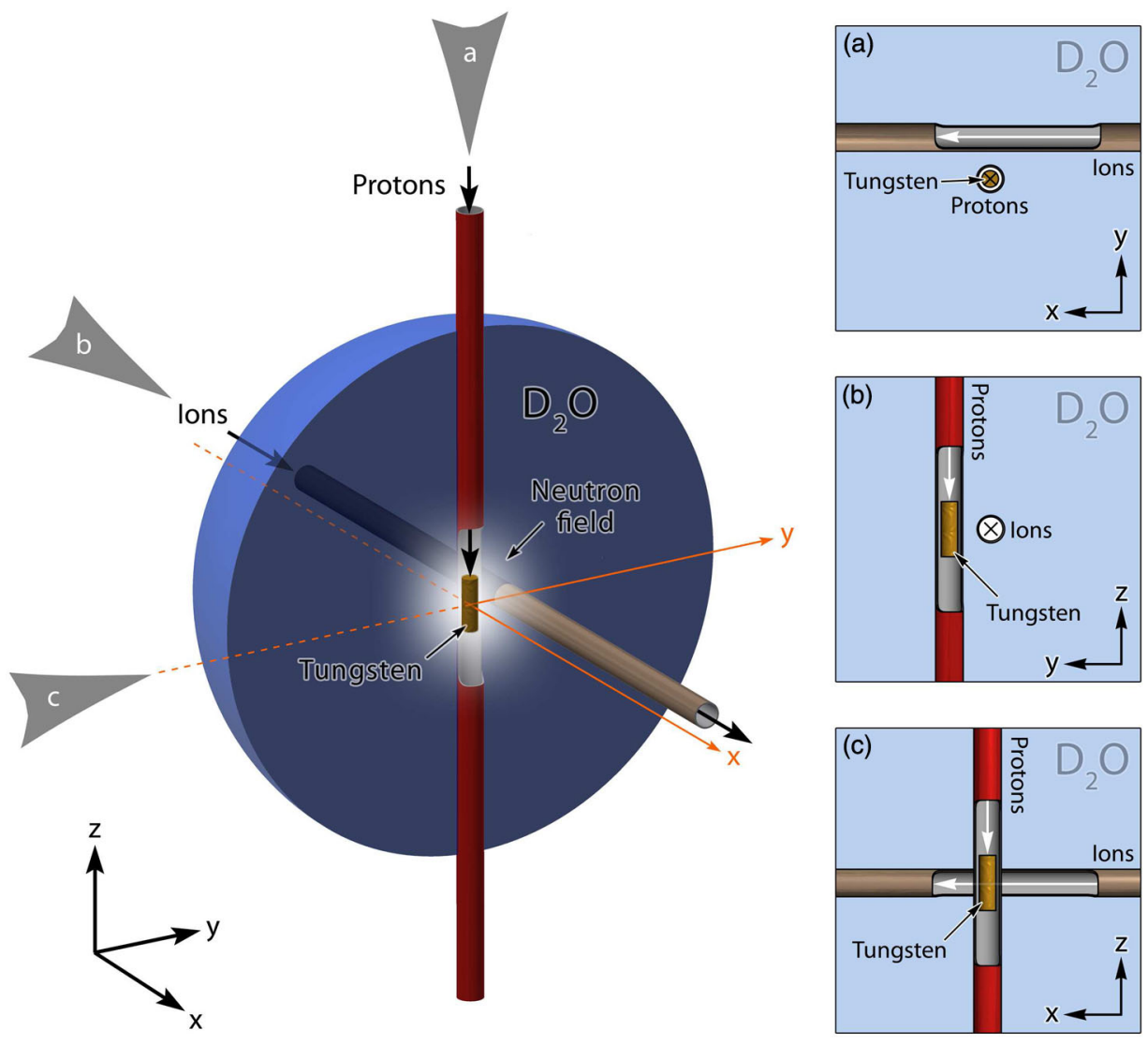

FIG. 2. Sketch of the proposed setup. Left: Protons impinge on a tungsten cylinder and produce neutrons. The tungsten spallation target (brown) inside the proton beam pipe (red) is shown in the center of the sketch. (In the sketch, the pipe is cut open to reveal the tungsten target.) The proton beam pipe points along the $\mathrm{z}$-axis and is orientated perpendicular to the ion beam pipe (gray). The beam pipes do not intersect as the ion beam pipe is shifted by several centimeters. The neutrons produced in the spallation process are moderated in the surrounding heavy water (blue, only half of the sphere is shown). They penetrate the ion beam pipe and act as a neutron target for the ions. The ends of ion beam pipe can be connected on the outside to form a storage ring. Right: 2D projections of the setup (a) along the proton beam pipe, (b) along the ion beam pipe, and (c) perpendicular to both pipes. The lines of sight are indicated by gray arrows in the left sketch. The water moderator is indicated by the light blue background. 
absorbed on the outside. Again, this will not alter the neutron budget.

\section{SIMULATIONS}

We simulated the proposed setup with GEANT-3.21 [7] with the GCALOR package [8]. As a first step, neutrons of different energies were emitted isotropically from the center of the tungsten target with the tungsten target in the center of the moderator (Sec. III A). These simulations help to understand the underlying principles and allow first rough estimates of the neutron density in the ion beam pipe. In addition, they can be used to estimate the effect of different primary neutron energies. In the second step, we simulated the interaction of a high-energy proton beam with a tungsten target (Sec. III B). All particles were followed until they either left the volume or were absorbed.

\section{A. Neutrons started with different energies}

Neutrons with different energies from $10^{-2}$ to $10^{9} \mathrm{eV}$ were randomly started in the tungsten spallation target for each moderator thickness (radii 0.5, 1.0, and $2.0 \mathrm{~m}$ ). The direction of the neutrons was chosen isotropically. A separate simulation for each energy decade was performed. The energy distribution within a decade was assumed to be $1 / E$ [5]. Each simulation run included $10^{6}$ neutrons.

The neutrons penetrate the ion beam pipe where they act as a neutron target for the ions. We investigated the average time period a neutron spends in the ion beam pipe. The average time period depends on the velocity of the neutron, the passing angle, and the number of times the neutron actually crosses the ion beam pipe. The time period spent inside the ion tube is summed up for each neutron emitted from the spallation target and stored into a histogram.

Figure 3 shows examples for neutrons of selected primary energy ranges. When the primary neutron energy increases, the number of events with at least one pass through the beam pipe increases as well as the number of crossings. In addition, very short time periods of a few $10^{-9} \mathrm{~s}$ are only observed for higher primary neutron energies. At lower energies even a single pass requires a few $10^{-6} \mathrm{~s}$.

Figure 4 shows the average time period a neutron spends in the ion beam pipe as a function of its original energy. The period is only weakly dependent on the original energy since the neutrons are quickly moderated and trapped inside the moderator until they finally escape. If the energy exceeds the neutron separation energy of deuterium, about 2.2 MeV, additional neutrons are available. Their time periods inside the beam pipe are added to the time of the original neutron. The larger the moderator volume, the longer the time period inside the ion beam pipe.

It is very important to note that the neutrons spend on average several microseconds in the ion beam pipe even for a relatively small moderator of only $50 \mathrm{~cm}$ in radius. A moderator of 2-m radius leads to a period of about

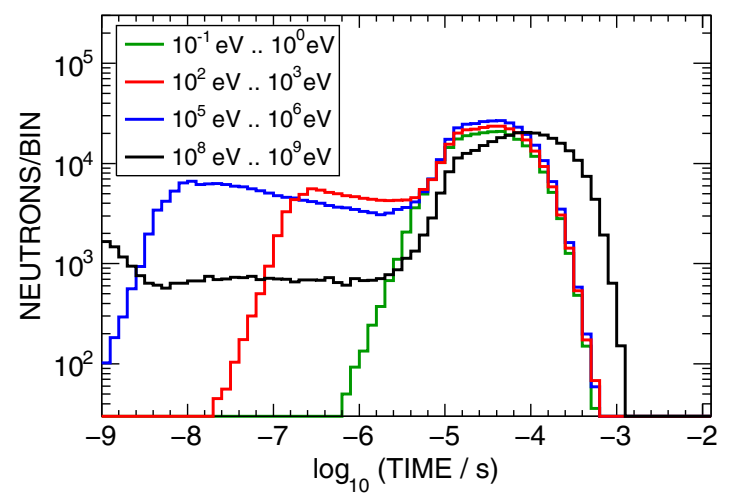

FIG. 3. Total time periods neutrons spend inside the ion beam pipe which is surrounded by a moderator of $1 \mathrm{~m}$ radius. The different histograms correspond to different primary neutron energy ranges, which were chosen exemplary from the entire neutron energy range from $10^{-2}$ to $10^{9} \mathrm{eV}$. For each energy decade, $10^{6}$ initial neutrons were simulated. Events where the neutron bypasses the ion beam pipe are not shown (zero suppressed). About $30 \%$ to $50 \%$ of all neutrons pass the ion beam pipe at least once. The $\mathrm{x}$-axis has a logarithmic binning with ten bins per time decade.

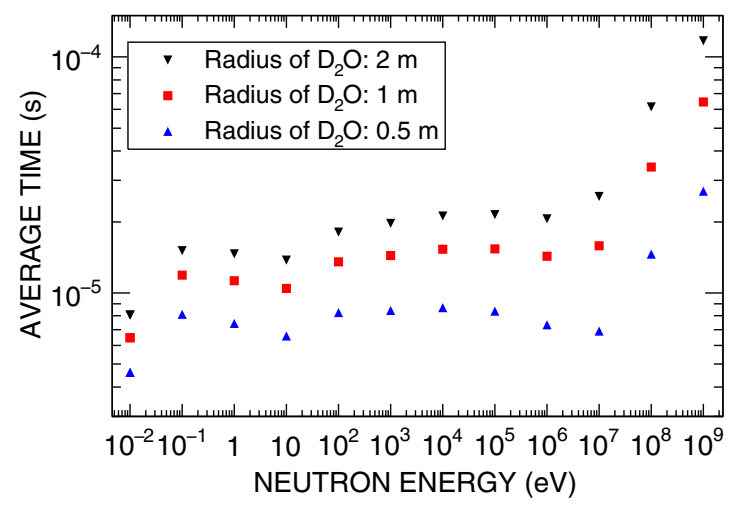

FIG. 4. Average time period a neutron spends inside the ion beam pipe for the different moderator sizes. The energy on the $\mathrm{x}$-axis corresponds to the lower edge of the energy interval. Each interval corresponds to one energy decade, hence, the point at $10^{8} \mathrm{eV}$ corresponds to neutron energies between $10^{8}$ and $10^{9} \mathrm{eV}$.

$20 \mu$ s. The longer the average time period inside the beam pipe, the higher the density of the neutron target for a given proton current.

\section{B. Protons energies of $800 \mathrm{MeV}$ and $20 \mathrm{GeV}$}

We simulated the setup with proton energies of $800 \mathrm{MeV}$ and $20 \mathrm{GeV}$. The chosen energies correspond to the energies the LANSCE accelerator at Los Alamos National Laboratory (800 MeV) [9] and the proton synchrotron at CERN $(20 \mathrm{GeV})[1,10]$ deliver. We investigated two different tungsten target sizes, a cylinder with a radius of $1.5 \mathrm{~cm}$ and a length of $10 \mathrm{~cm}$, and one with radius of $2.5 \mathrm{~cm}$ and a length of $50 \mathrm{~cm}$. The four different simulation settings are listed in Table I. The table also gives the neutron yield per proton. 
TABLE I. Neutron yield for the four different settings.

\begin{tabular}{lcc}
\hline \hline Proton energy & $\begin{array}{c}\text { W-target } \\
\text { radius } \times \text { length } \\
(\mathrm{GeV})\end{array}$ & Neutrons/proton $)$ \\
\hline 0.8 & $1.5 \times 10$ & 11.8 \\
0.8 & $2.5 \times 50$ & 15.8 \\
20 & $1.5 \times 10$ & 70.1 \\
20 & $2.5 \times 50$ & 227 \\
\hline \hline
\end{tabular}

Figure 5 shows the energy spectra of the produced neutrons for all simulated combinations of target size and proton energy. In general, the number of spallation neutrons increases with the proton energy. As the $20-\mathrm{GeV}$ protons will not be slowed down below the spallation threshold within $10 \mathrm{~cm}$ of tungsten, the neutron yield is significantly higher for the larger tungsten target.

Each proton produces a certain amount of neutrons (see Table I). These neutrons travel through the setup, are moderated by the heavy water, and eventually pass the ion beam pipe. The total time period $t_{\text {neutron,tot }}$ sums up the time periods that all neutrons produced by a proton spend inside the beam pipe. Averaging over all protons, we obtain the average total time period that all neutrons produced by a proton spend inside the ion beam tube $\bar{t}_{\text {neutron,tot }}$. To estimate the average number of neutrons inside the beam tube, $\bar{n}_{\text {neutron}}$, we multiply $\bar{t}_{\text {neutron,tot }}$ by the number of protons hitting the spallation target per time (proton current/elementary charge):

$$
\bar{n}_{\text {neutron }}=\frac{I_{\text {proton }}}{e} \bar{t}_{\text {neutron,tot }} \text {. }
$$

Figure 6 shows $t_{\text {neutron,tot }}$ for primary protons with different energies and tungsten target sizes, but without a moderator. The averages of the distributions correspond to $\bar{t}_{\text {neutron,tot. }}$. Protons with an energy of $20 \mathrm{GeV}$ produce more neutrons (see Table I). Hence, the total time period the

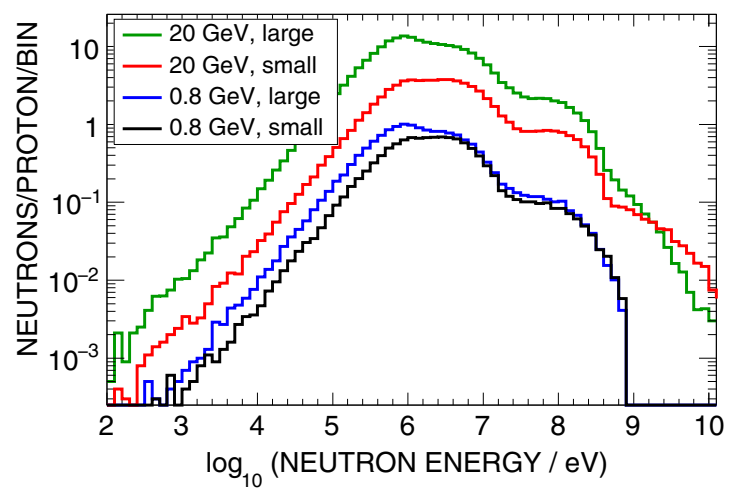

FIG. 5. Spallation neutron spectra for the different settings, see also Table I. The x-axis has a logarithmic binning with ten bins per energy decade.

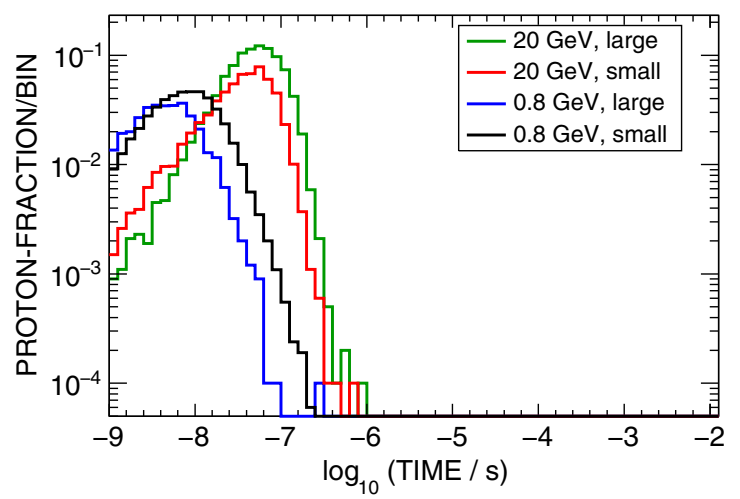

FIG. 6. Proton fraction as a function of the total time period the produced neutrons spend in the ion beam pipe. The simulations were carried out without a moderator. The distributions are shown for different proton energies and tungsten target sizes (see also Table II and the discussion of Eq. (1)). The x-axis has a logarithmic binning with ten bins per time decade.

neutrons spend inside the beam pipe is higher compared to the $800-\mathrm{MeV}$ protons. The $20-\mathrm{GeV}$ protons produce about a factor of 3 more neutrons in the large tungsten target than in the small one. The $800-\mathrm{MeV}$ protons produce only slightly more neutrons in the large target. But then the larger tungsten target acts as a neutron trap. The larger neutron production outweighs the neutron captures only in the case of $20-\mathrm{GeV}$ protons, but not in the case of $800-\mathrm{MeV}$ protons. Therefore, the total time period the produced neutrons spend in the ion beam pipe is shifted to smaller values for $800-\mathrm{MeV}$ protons impinging on the large target.

If a moderator is included, the total time period neutrons spend inside the ion beam pipe changes dramatically. Figure 7 shows the results for a moderator with a radius of $1 \mathrm{~m}$. The averaged time period all neutrons produced per incident proton spend inside the ion beam pipe $\left(\bar{t}_{\text {neutron,tot }}\right)$ is listed in Table II for all combinations of proton energy, tungsten target size and moderator radius.

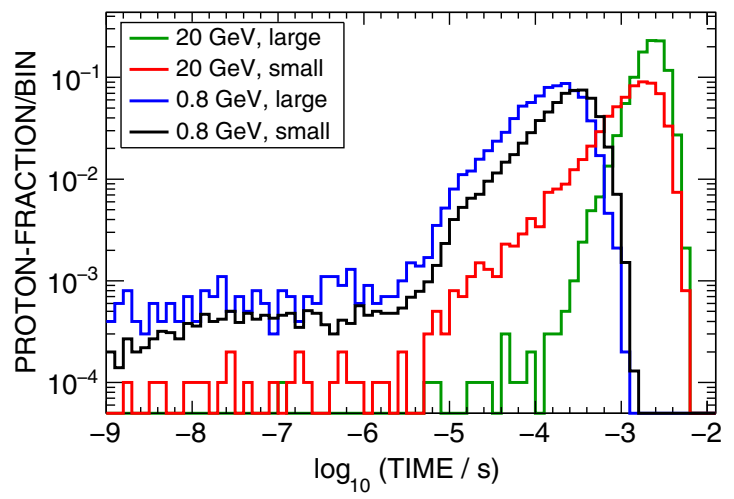

FIG. 7. The distribution of the total time period that all neutrons produced by a single proton spend inside the ion beam pipe for a moderator $1 \mathrm{~m}$ in radius and all target/beam combinations listed in Table II and the discussion of Eq. (1). The X-axis has a logarithmic binning with ten bins per time decade. 
TABLE II. Average total time period neutrons spend inside the ion beam pipe per proton $\left[\bar{t}_{\text {neutron,tot }}\right.$, see Eq. (1)]. The recommended settings for a beam energy of $800 \mathrm{MeV}$ and $20 \mathrm{GeV}$ are printed in bold.

\begin{tabular}{lccc}
\hline \hline $\begin{array}{l}\text { Proton } \\
\text { energy } \\
(\mathrm{GeV})\end{array}$ & $\begin{array}{c}\text { W-target } \\
\text { radius } \times \text { length } \\
(\mathrm{cm} \times \mathrm{cm})\end{array}$ & $\begin{array}{c}\text { Moderator } \\
\text { radius } \\
(\mathrm{m})\end{array}$ & $\begin{array}{c}\text { Time } \\
(\mu \mathrm{s})\end{array}$ \\
\hline 0.8 & $1.5 \times 10$ & 0.0 & 0.0051 \\
0.8 & $1.5 \times 10$ & 0.5 & 81 \\
0.8 & $1.5 \times 10$ & 1.0 & 162 \\
$\mathbf{0 . 8}$ & $\mathbf{1 . 5} \times \mathbf{1 0}$ & $\mathbf{2 . 0}$ & $\mathbf{2 4 5}$ \\
0.8 & $2.5 \times 50$ & 0.0 & 0.0022 \\
0.8 & $2.5 \times 50$ & 0.5 & 62 \\
0.8 & $2.5 \times 50$ & 1.0 & 142 \\
0.8 & $2.5 \times 50$ & 2.0 & 224 \\
20 & $1.5 \times 10$ & 0.0 & 0.028 \\
20 & $1.5 \times 10$ & 0.5 & 490 \\
20 & $1.5 \times 10$ & 1.0 & 1000 \\
20 & $1.5 \times 10$ & 2.0 & 1560 \\
20 & $2.5 \times 50$ & 0.0 & 0.057 \\
20 & $2.5 \times 50$ & 0.5 & 1060 \\
20 & $2.5 \times 50$ & 1.0 & 2260 \\
$\mathbf{2 0}$ & $\mathbf{2 . 5} \times \mathbf{5 0}$ & $\mathbf{2 . 0}$ & $\mathbf{3 4 7 0}$ \\
\hline \hline
\end{tabular}

Figure 8 shows the position distribution of neutrons inside the ion beam pipe. For this plot, the beam axis was subdivided into disks of $1 \mathrm{~cm}$ width in the direction of the beam. Whenever a neutron entered one of the disks, it was recorded in the histogram. The fact that high-energy neutrons do not spend much time inside the beam pipe is considered in Fig. 9, where each entry was weighted with the corresponding time the neutron spends in the disk. From the comparison of Figs. 8 and 9 one finds that the suppressed high-energy neutrons, which are usually coming directly from the spallation target, can be found mostly in the center of the ion beam pipe, close to the proton beam pipe.

Figure 10 shows the energy distribution of the neutrons in the ion beam pipe, the small tungsten target, $800-\mathrm{MeV}$

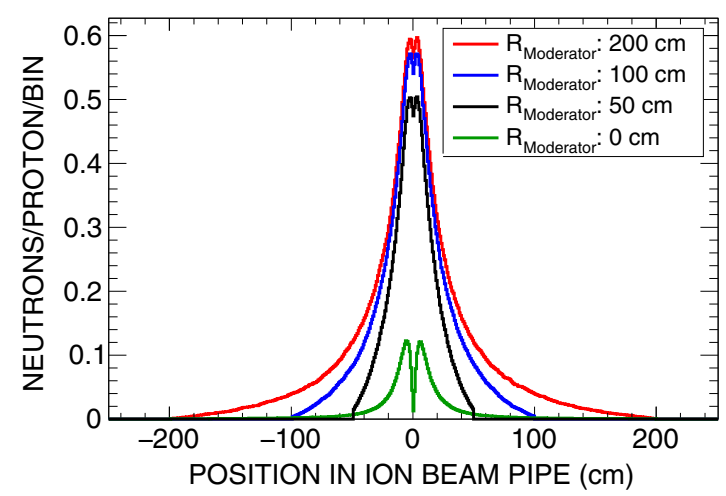

FIG. 8. Neutron position along the ion beam for different moderators, the small version of the tungsten target and $800-\mathrm{MeV}$ protons. The $\mathrm{x}$-axis has a bin width of $1 \mathrm{~cm}$.

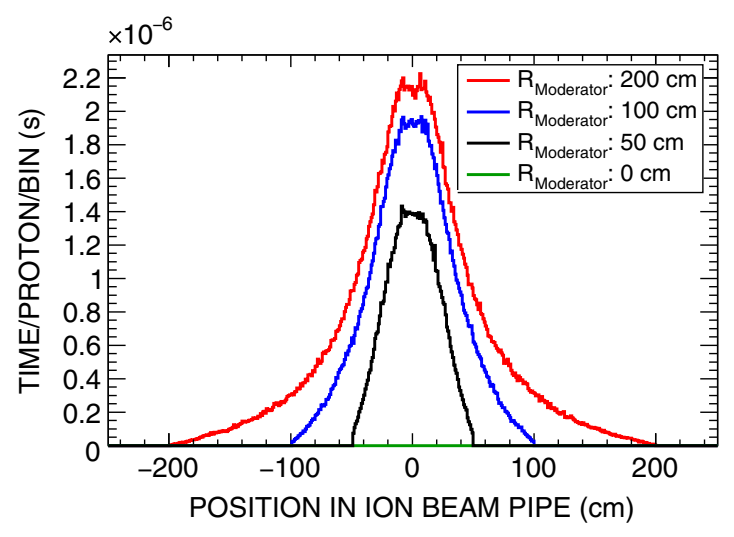

FIG. 9. The total time period neutrons spend inside the ion beam pipe as a function of position along the ion beam for different moderators, the small tungsten target and $800 \mathrm{MeV}$ protons. Each entry corresponds to the sum of neutrons weighted with the time period the neutron has spent inside the tube. The $\mathrm{x}$-axis has a bin width of $1 \mathrm{~cm}$.

protons and different moderator sizes. Without a moderator, the energy distribution resembles the spallation neutron spectrum, compare Fig. 5. The moderation process produces neutron spectra with a thermal energy distribution. The larger the moderator, the more neutrons per proton enter the ion beam pipe. Figure 11 shows the energy spectra are weighted with the corresponding time period the neutron spent inside the ion beam pipe. High-energy neutrons pass the pipe quickly. The moderated neutrons spend up to $2 \mu \mathrm{s}$ in the pipe.

As can be seen from Figs. 10 and 11, the neutron spectrum in the ion beam pipe is clearly dominated by lowenergy neutrons. Therefore, the center-of-mass energy of the ion-neutron collision in realistic experiments will be defined by the energy of the ions. The ion energy can easily be tuned in the storage ring by applying, for instance, electron cooling at a defined beam energy.

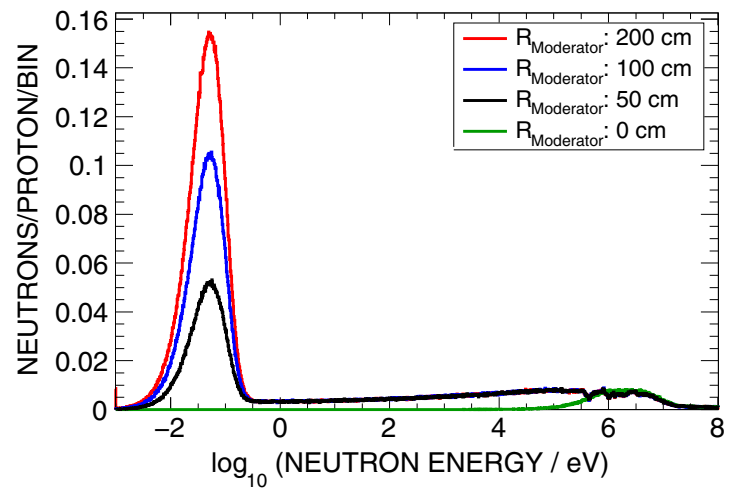

FIG. 10. Energy distribution of all neutrons entering the ion beam pipe for different moderator sizes, the small tungsten target and $800-\mathrm{MeV}$ protons. The $\mathrm{x}$-axis has a logarithmic binning with 100 bins per energy decade. 


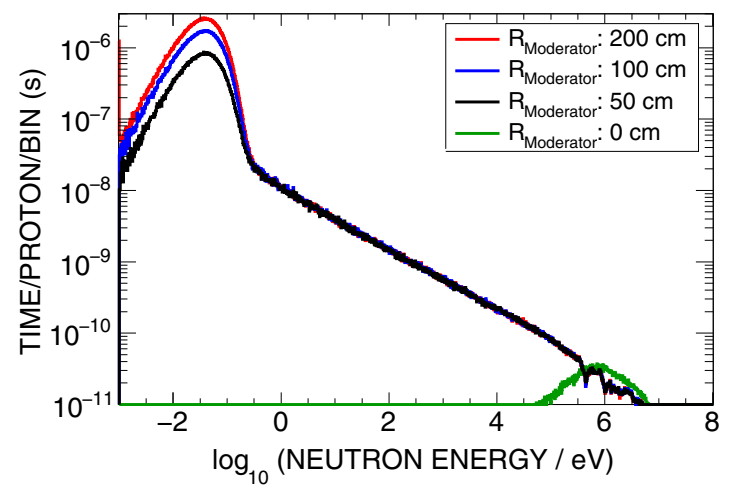

FIG. 11. Total time period neutrons spend inside the ion beam pipe as a function of the neutron energy for different moderator sizes, the small tungsten target and $800-\mathrm{MeV}$ protons. Each entry corresponds to the sum of neutrons weighted with the time period the neutron spent inside the ion tube. The $\mathrm{x}$-axis has a logarithmic binning with 100 bins per energy decade.

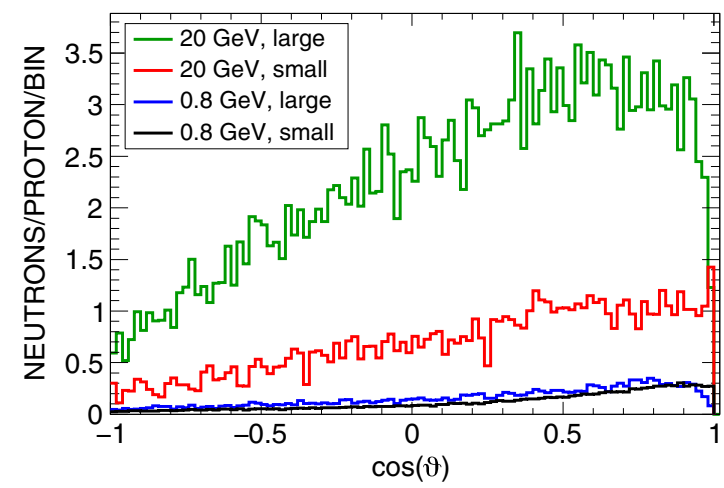

FIG. 12. Neutron emission angle from the spallation target with respect to the direction of the protons for all settings. The $\mathrm{x}$-axis has 100 bins.

The simulations were repeated with a different tungsten target position relative to the moderator. We moved the target upstream since most of the neutrons are emitted in the direction of the impinging high-energy proton beam (Fig. 12), potentially gaining more forward-emitted neutrons. However, the average time period neutrons spend inside the ion beam pipe was reduced. The scattering processes in the moderator alters the original direction of the neutron quickly. Hence, the emission angle of the neutrons is not important.

\section{POSSIBLE REALIZATIONS}

We discuss possible realizations of the proposed setup at different experimental facilities. We consider the available proton currents and energies to estimate the number of neutrons inside an ion beam tube running through a heavywater moderator. We estimate the average time period neutrons spend inside the ion beam tube per incoming proton from the proton energy, Table II and Eq. (1). We calculate the areal neutron density $\eta_{\text {neutron }}$ by dividing the average number of neutrons inside the beam tube $\bar{n}_{\text {neutron }}$ by the cross section of the ion beam pipe $A_{\text {ion pipe: }}$ :

$$
\eta_{\text {neutron }}=\frac{\bar{n}_{\text {neutron }}}{A_{\text {ion pipe }}}=\frac{I_{\text {proton }}}{e} \frac{\bar{t}_{\text {neutron,tot }}}{A_{\text {ion pipe }}} .
$$

The set of simulations described here was driven by the specific opportunities of the LANSCE-accelerator at LANL and the PS at CERN. These facilities have already wellestablished spallation neutron sources with the corresponding driving accelerators. Therefore, they are currently the most promising options. However, the idea of using a heavily moderated spallation source in conjunction with a storage ring is certainly not restricted to these facilities, but may be set up at other institutions like GSI/FAIR, HIRFL, HIAF, NSCL/MSU, MYRRHA. In particular, new developments in cyclotron technology like fixed field alternating gradient machines [11] offer the possibility to realize this setup at a dedicated facility.

\section{A. Los Alamos National Laboratory (LANL)}

The Lujan target at LANSCE/LANL operates with an average proton current of about $100 \mu \mathrm{A}$. We consider an ion beam tube with a cross section of $20 \mathrm{~cm}^{2}$. We obtain a neutron density of about $8 \times 10^{9} \mathrm{n} / \mathrm{cm}^{2}$ using the largest moderator with a diameter of $200 \mathrm{~cm}$. The neutron density is only a factor of 2 less than described in Ref. [6], where a neutron flux of $10^{14} \mathrm{n} / \mathrm{cm}^{2} / \mathrm{s}$ in a reactor and an interaction length of $0.5 \mathrm{~m}$ was assumed. The results for the different moderator sizes are given in Table III.

The LANSCE accelerator provides $\mathrm{H}^{-}$and $\mathrm{H}^{+}$ions $[9,12]$. A magnet bends $\mathrm{H}^{-}$ions to the southern experimental areas, and $\mathrm{H}^{+}$ions to the northern area. Additional facilities, like the proposed combination of an ion storage ring and a neutron target, could be installed at the northern area. Here, $\mathrm{H}^{+}$beams with currents up to $1 \mathrm{~mA}$ could be delivered. In particular, the nearby isotope production facility, located at the beginning of the LANSCE accelerator, makes this idea very attractive. The required amount of radioactive material for an experiment in inverse

TABLE III. Neutron density for the simulated setup at two facilities: $100 \mu \mathrm{A}$ proton beam with $800 \mathrm{MeV}$ and the small tungsten target (LANL), as well as $3 \times 10^{12}$ protons/s with an energy of $20 \mathrm{GeV}$ and the large tungsten target (CERN), see Eq. (2).

\begin{tabular}{lcc}
\hline \hline & \multicolumn{2}{c}{ Neutron density $\left(\mathrm{cm}^{-2}\right)$} \\
\cline { 2 - 3 } Moderator radius $(\mathrm{m})$ & LANL & CERN \\
\hline 0.0 & $1.6 \times 10^{6}$ & $8.7 \times 10^{3}$ \\
0.5 & $2.6 \times 10^{9}$ & $1.6 \times 10^{8}$ \\
1.0 & $5.2 \times 10^{9}$ & $3.6 \times 10^{8}$ \\
2.0 & $7.8 \times 10^{9}$ & $5.4 \times 10^{8}$ \\
\hline \hline
\end{tabular}


kinematics is orders of magnitude smaller than the amount of material needed for a traditional time-of-flight experiment like DANCE $[2,13,14]$. Typically, the storage ring needs to be filled every few minutes with about $10^{7}$ ions [15] consuming about $1.5 \times 10^{10}$ atoms during a day.

\section{B. European Organization for Nuclear Research (CERN)}

The n_TOF/ISOLDE experiments receive about $3 \times 10^{12}$ protons/s with an energy of $20 \mathrm{GeV}$. On average, these protons could produce between $1.5 \times 10^{9}$ and $10^{10}$ neutrons in an ion beam pipe as described here. The resulting neutron densities of up to $6 \times 10^{8} \mathrm{n} / \mathrm{cm}^{2}$ in the pipe are listed in Table III. The installation of the wellsuited ion storage ring TSR at HIE-ISOLDE [16] would provide the main ingredients of the proposed setup.

\section{GSI Helmholtz Center for Heavy Ion Research (GSI) and Facility for Antiproton and Ion Research (FAIR)}

The universal linear accelerator UNILAC and the 18-T $\mathrm{m}$ heavy-ion synchrotron SIS-18 are the driver accelerators at GSI in Darmstadt (Germany). Proton intensities of up to $2.1 \times 10^{11}$ protons per pulse have routinely been achieved [17]. The magnetic rigidity of $18 \mathrm{~T} \mathrm{~m}$ allows one to accelerate protons to energies of $4.5 \mathrm{GeV}$. The $100-\mathrm{T} \mathrm{m}$ synchrotron of the future FAIR facility [18] will provide about $5 \times 10^{12}$ protons/s with an energy of $28.8 \mathrm{GeV}$.

A variety of specialized facilities like fragment separators [19,20], storage rings [21,22], experimental caves, and beam lines [23,24] provides numerous opportunities to realize a low-energy storage ring combined with a spallation target described here.

\section{Heavy Ion Research Facility in Lanzhou (HIRFL) and Cooler Storage Ring (CSR) Complex (IMP) and High Intensity Heavy-Ion} Accelerator Facility (HIAF)

The Heavy Ion Research Facility in Lanzhou (HIRFL), China, which is similar to GSI, has been operational since 2007 [25]. The maximum magnetic rigidity of the main synchrotron ring is $10.6 \mathrm{~T} \mathrm{~m}$ (maximum proton energy is $2.3 \mathrm{GeV}$ ). The existing cooler storage ring CSRe might be employed to store radioactive ions. The High Intensity Heavy-Ion Accelerator Facility (HIAF) has been approved and will be constructed in Huizhou, China. The considered rigidity of the main synchrotron will be around $30 \mathrm{~T} \mathrm{~m}$, thus providing about $8 \mathrm{GeV}$ protons. The beam intensities are comparable to those expected at FAIR. Several high-energy as well as low-energy storage rings are being considered for HIAF. Here, the proposed combination of a storage ring with a spallation target could be considered already at the planning stage of the facility. Furthermore, a high power 1.0 to $1.5 \mathrm{GeV}$ dedicated proton linear accelerator is under construction at the same location as HIAF within the running accelerator-driven systems (ADS) project in China.

\section{CONCLUSIONS}

The combination of an intense neutron source and an ion storage ring would be unique for a direct measurement of neutron-induced reactions, as already discussed in [6]. Direct kinematics, where the neutrons impinge on the target of interest, require the measurement of the light reaction products. The $\gamma$-rays from the investigated reaction have to be discriminated from the sample decay $\gamma$-rays. The proposed technique is based on the detection of the heavy, projectile-like residues of the reactions, which is a significant advantage for (a) the measurement of $(n, \gamma)$ cross sections of $\gamma$-emitting samples or of fissile nuclei, (b) the measurement of $(n, p)$ and $(n, \alpha)$ cross sections at low energies, as there is no need to detect the emitted protons and $\alpha$-particles, and (c) for cross section measurements on relatively long-lived or even stable nuclei.

\section{SUMMARY}

A typical spallation neutron source can be modified to build a neutron target by combination with a large moderator of heavy water. The resulting neutron target, intercepted by an ion beam, can be used to investigate neutron-induced reactions. This technique is of advantage if the sample size in a traditional time-of-flight setup is limited, either because of the decay properties of the investigated isotope or because of the range of the reaction products. In particular, the combination with a storage ring makes this technique feasible. Existing facilities like LANSCE at LANL, n_TOF/ ISOLDE at CERN, or GSI/FAIR could be complemented with the setup described here.

\section{ACKNOWLEDGMENTS}

This research has received funding from the European Research Council under the European Union's Seventh Framework Programme (FP/2007-2013)/ERC Grant Agreement No. 615126. We thank Hushan Xu for his valuable information about the planned spallation sources in China (ADS and HIAF).

[1] R. Reifarth, C. Lederer, and F. Käppeler, Neutron reactions in astrophysics, J. Phys. G 41, 053101 (2014).

[2] A. Couture and R. Reifarth, Direct measurements of neutron capture on radioactive isotopes, At. Data Nucl. Data Tables 93, 807 (2007).

[3] M. Heil, R. Reifarth, M. M. Fowler, R. C. Haight, F. Käppeler, R. S. Rundberg, E. H. Seabury, J. L. Ullmann, J. B. Wilhelmy, K. Wisshak, and F. Voss, A $4 \pi \mathrm{BaF}_{2}$ detector for $(\mathrm{n}, \gamma)$ cross-section measurements at a spallation neutron source, Nucl. Instrum. Methods Phys. Res., Sect. A 459, 229 (2001). 
[4] R. Reifarth, R. C. Haight, M. Heil, F. Käppeler, and D. J. Vieira, Neutron capture measurements at a RIA-type facility, Nucl. Instrum. Methods Phys. Res., Sect. A 524, 215 (2004).

[5] R. Reifarth, T. A. Bredeweg, A. Alpizar-Vicente, J. C. Browne, E.-I. Esch, U. Greife, R. C. Haight, R. Hatarik, A. Kronenberg, J. M. O'Donnell, R. S. Rundberg, J. L. Ullmann, D. J. Vieira, J. B. Wilhelmy, and J. M. Wouters, Background identification and suppression for the measurement of $(n, \gamma)$ reactions with the DANCE array at LANSCE, Nucl. Instrum. Methods Phys. Res., Sect. A 531, 530 (2004).

[6] R. Reifarth and Y. A. Litvinov, Measurements of neutroninduced reactions in inverse kinematics, Phys. Rev. ST Accel. Beams 17, 014701 (2014).

[7] J. Apostolakis, CERN program library long writeup, W5013, Technical Report, CERN, GEANT library, 1993.

[8] C. Zeitnitz and T. Gabriel, The GEANT-CALOR interface and benchmark calculations of ZEUS test calorimeters, Nucl. Instrum. Methods Phys. Res., Sect. A 349, 106 (1994).

[9] P. W. Lisowski, C. D. Bowman, G. J. Russell, and S. A. Wender, The Los Alamos National Laboratory spallation neutron sources, Nucl. Sci. Eng. 106, 208 (1990).

[10] C. Guerrero et al., Performance of the neutron time-offlight facility n_TOF at CERN, Eur. Phys. J. A 49, 27 (2013).

[11] L. Calabretta and F. Méot, Cyclotrons and FFAG Accelerators as Drivers for ADS, Rev. Accel. Sci. Techol. 08, 77 (2015).

[12] A. Michaudon and S. Wender, Los Alamos National Laboratory Technical Report No. LA-UR-90-4355, 1990, http://permalink.lanl.gov/object/tr?what=info: lanl-repo/lareport/LA-UR-90-4355.

[13] E.-I. Esch et al., Measurement of the ${ }^{237} \mathrm{~Np}(n, \gamma)$ cross section from $20 \mathrm{meV}$ to $500 \mathrm{keV}$ with a high efficiency, highly segmented $4 \pi \mathrm{BaF}_{2}$ detector, Phys. Rev. C 77, 034309 (2008).

[14] M. Weigand, T. A. Bredeweg, A. Couture, K. Göbel, T. Heftrich, M. Jandel, F. Käppeler, C. Lederer, N. Kivel,
G. Korschinek, M. Krtička, J. M. O’Donnell, J. Ostermöller, R. Plag, R. Reifarth, D. Schumann, J. L. Ullmann, and A. Wallner, ${ }^{63} \mathrm{Ni}(n, \gamma)$ cross sections measured with DANCE, Phys. Rev. C 92, 045810 (2015).

[15] B. Mei et al., First measurement of the ${ }^{96} \mathrm{Ru}(p, \gamma){ }^{97} \mathrm{Rh}$ cross section for the $p$ process with a storage ring, Phys. Rev. C 92, 035803 (2015).

[16] M. Grieser et al., Storage ring at HIE-ISOLDE, Eur. Phys. J. Spec. Top. 207, 1 (2012).

[17] W. Barth, A. Adonin, S. Appel, P. Gerhard, M. Heilmann, F. Heymach, R. Hollinger, W. Vinzenz, H. Vormann, and S. Yaramyshev, Heavy ion linac as a high current proton beam injector, Phys. Rev. ST Accel. Beams 18, 050102 (2015).

[18] H. H. Gutbrod, I. Augustin, H. Eickhoff, K.-D. Groß, W. F. Henning, D. Krämer, and G. Walter, FAIR Baseline Technical Report (GSI, 2006), ISBN 3-9811298-0-6.

[19] H. Geissel et al., The GSI projectile fragment separator (FRS): A versatile magnetic system for relativistic heavy ions, Nucl. Instrum. Methods Phys. Res., Sect. B 70, 286 (1992).

[20] H. Geissel et al., The Super-FRS project at GSI, Nucl. Instrum. Methods Phys. Res., Sect. B 204, 71 (2003).

[21] B. Franzke, The heavy ion storage and cooler ring project ESR at GSI, Nucl. Instrum. Methods Phys. Res., Sect. B 24-25, 18 (1987).

[22] M. Lestinsky et al., Physics book: CRYRING@ESR, Eur. Phys. J. Spec. Top. 225, 797 (2016).

[23] F. Bosch, Y. A. Litvinov, and T. Stöhlker, Nuclear physics with unstable ions at storage rings, Prog. Part. Nucl. Phys. 73, 84 (2013).

[24] T. Stöhlker, Y. A. Litvinov, A. Bräuning-Demian, M. Lestinsky, F. Herfurth, R. Maier, D. Prasuhn, R. Schuch, and M. Steck, SPARC collaboration: New strategy for storage ring physics at FAIR, Hyperfine Interact. 227, 45 (2014).

[25] J. Xia et al., The heavy ion cooler-storage-ring project (HIRFL-CSR) at Lanzhou, Nucl. Instrum. Methods Phys. Res., Sect. A 488, 11 (2002). 